\title{
Influence of the coating on the corrosion rate in concrete beams exposed to environment -DURACON Project
}

\author{
M.A.B. Zamora, ${ }^{1,{ }^{*}}$ D.N. Mendoza, ${ }^{1}$ H.H. Zamora, ${ }^{2}$ F.A. Calderón ${ }^{3}$ \\ ${ }^{1}$ Facultad de Ingeniería Civil - Xalapa, Universidad Veracruzana, \\ Circ. G. Aguirre Beltrán S/N, Xalapa, Veracruz, México, CP 91090 \\ ${ }^{2}$ Facultad de Ingeniería Industrial. UPA. Altamira, Tamaulipas, México. \\ ${ }^{3}$ CIMAV S.C. Miguel de Cervantes 120 Chihuahua, Chih. México
}

Received 25 April 2008; accepted 23 October 2008

\begin{abstract}
This work is part of the DURACON iberoamerican project, which deals with the characterization of the concrete durability exposed to environmental conditions at Iberoamerica, through the exposure of reinforced concrete specimens in at least two different environments, one marine and one urban, in each participating country. In this paper, results of the first eight months of the specimen exposition to environmental conditions in the Xalapa city, Mexico, are evaluated. The specimens were built with two types of concretes at 0.45 and 0.65 water/cement ratios. In all cases, the same type of cement with three thicknesses $(1.5,2.0$ and $3.0 \mathrm{~cm})$ of covering were used. The tests to evaluate the susceptibility to corrosion of the reinforced steel were resistance to linear polarization and monitoring of the corrosion potentials according to the ASTM C-876 norm.
\end{abstract}

Keywords: durability, corrosion, concrete, environment, coating.

\section{Introducción}

Hasta hace unos años se pensaba que las estructuras de hormigón hidráulico tenían una vida útil ilimitada y que su durabilidad dependía únicamente de sus características mecánicas, por lo que, durante la etapa de su diseño, no se tomaban en cuenta las condiciones ambientales a las cuales iba a estar expuestas. Hoy día, diferentes investigadores han demostrado que la durabilidad de dichas estructuras no solo depende de sus características mecánicas, sino también del medio ambiente en el que van a estar inmersas [1]. Uno de los estudios más recientes realizados para relacionar la durabilidad de las estructuras de hormigón con el medio ambiente, es el realizado por el grupo DURACON (Influencia del medio ambiente en la durabilidad del hormigón), a través del proyecto del mismo

\footnotetext{
* Corresponding author. E-mail address: mbaltazar@uv.mx
} 
nombre, auspiciado por el Programa de Ciencia y Tecnología para el Desarrollo (CYTED). Este proyecto está enfocado a estudiar el comportamiento del hormigón hidráulico expuesto a diferentes condiciones ambientales de Ibero América. En México se tienen 14 estaciones de monitorización. Una de ellas es la estación Xalapa, en el Estado de Veracruz, México, la cual acaba de cumplir 8 meses de estudio. El objetivo de este trabajo es presentar los resultados preliminares de estos primeros meses de exposición de las vigas a un medio ambiente catalogado como urbano.

\section{Metodología experimental}

\section{Materiales para la fabricación de vigas de hormigón.}

Los especimenes bajo estudio fueron fabricados tomando en cuenta las siguientes especificaciones:

1. Tipo de cemento: Cemento Pórtland Ordinario [2].

2. Agregado silíceo triturado de $19 \mathrm{~mm}$ de tamaño máximo, densidad de 2.58.

3. Arena cuarzosa, $\mathrm{Ss}=2.50$.

4. Relación a/c: $0.45\left(\mathrm{f}^{\prime} \mathrm{c}=350 \mathrm{~kg} / \mathrm{cm}^{2}\right)$.

5. Relación a/c: $0.65\left(\mathrm{f}^{\prime} \mathrm{c}=210 \mathrm{~kg} / \mathrm{cm}^{2}\right)$.

6. Adición de plastificante $0.3 \%$ por peso de cemento (sikament $190 \mathrm{CR}$ ).

7. Revenimiento $10 \mathrm{~cm} \pm 1.0 \mathrm{~cm}$, cimbra metálica y curado por inmersión en agua durante 7 días.

Las proporciones para las diferentes mezclas se muestran en la Tabla 1.

Tabla 1. Proporciones de las mezclas empleadas.

\begin{tabular}{|c|c|c|}
\hline Contenido $\left(\mathbf{k g} / \mathbf{m}^{\mathbf{3}}\right)$ & Relación $\mathbf{0 . 4 5}$ & Relación $\mathbf{0 . 6 5}$ \\
\hline Cemento & 411 & 285 \\
\hline Agua & 185 & 185 \\
\hline Agregado pétreo & 1010 & 1033 \\
\hline Agregado fino & 731 & 812 \\
\hline Aditivo (plastificante) & \multicolumn{2}{|c|}{$4 \mathrm{cc} / \mathrm{kg}$ cemento } \\
\hline
\end{tabular}

\section{Preparación y colocación de las vigas}

Para cada estación fueron preparadas doce vigas de hormigón de $15 \mathrm{~cm} \mathrm{x} 15 \mathrm{~cm}$ x $30 \mathrm{~cm}$, de las cuales seis fueron de hormigón simple y las otras seis de hormigón reforzado con seis varillas de acero del \#3 (9.5 $\mathrm{mm}$ de diámetro). Las varillas fueron colocadas dentro de las vigas, con tres espesores diferentes de recubrimiento de hormigón: 15, 20 y $30 \mathrm{~mm}$. La Fig. 1 muestra un croquis de la geometría de las vigas del proyecto. La Fig. 2 muestra una fotografía de la estación de ensayo natural. Las vigas fueron colocadas en soportes metálicos, con una cara orientada hacia los vientos predominantes (cara expuesta) y la otra cara hacia los vientos no predominantes (cara resguardada) 


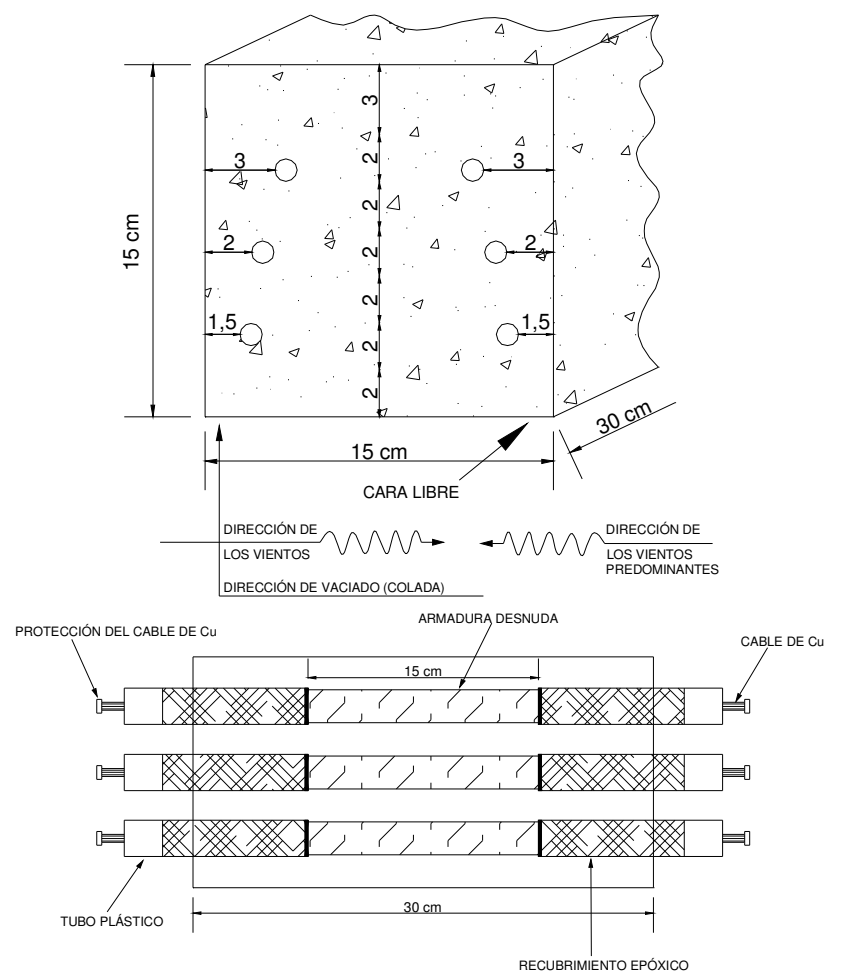

Figura 1. Croquis de la sección transversal y longitudinal de las vigas de hormigón reforzado del proyecto DURACON.

Las vigas de hormigón reforzado fueron empleadas para realizar la evaluación de los parámetros electroquímicos: Potencial de Corrosión $\left(\mathrm{E}_{\mathrm{corr}}\right)$ y cinética de corrosión $\left(\mathrm{i}_{\text {corr }}\right)$. La $\mathrm{i}_{\text {corr }}$ fue medida usando la técnica de Resistencia a la Polarización Lineal (Rp), de acuerdo con la norma ASTM G 59 - 97[3] y con la Red DURAR [4].

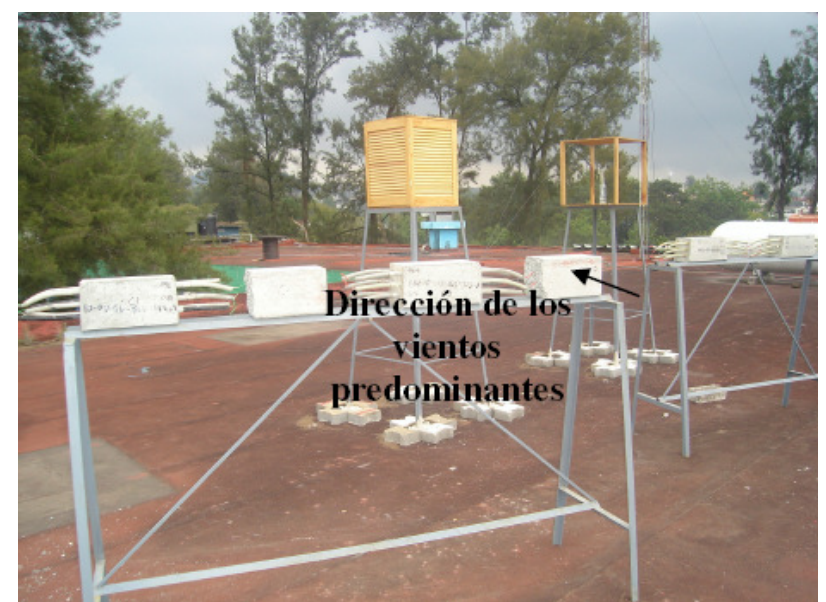

Figura 2. Estación de ensayo natural, orientada hacia los vientos predominantes.

\section{Resultados y discusión}

\section{Propiedades físicas de los hormigones}

En la Tabla 2 se presentan las propiedades físicas de los hormigones usados: porosidad efectiva $(\rho)$, coeficiente de absorción capilar $(\mathrm{ka})$, resistencia a la 
penetración de agua (m) y resistencia a la compresión (f'c) [5], La resistencia a la compresión obtenida fue mayor a la resistencia diseñada.

Tabla 2. Propiedades físicas de los hormigones empleados.

\begin{tabular}{|c|c|c|}
\hline Parámetro & Relación 0.45 & Relación 0.65 \\
\hline $\mathrm{f}^{\prime} \mathrm{c}, \mathrm{N} / \mathrm{mm}^{2}$ & 51.5 & 40.0 \\
\hline $\mathrm{m}, \mathrm{s} / \mathrm{m}^{2}$ & $3.48 \times 10^{7}$ & $3.60 \times 10^{7}$ \\
\hline $\mathrm{Ka}, \mathrm{kg} / \mathrm{m}^{2} \mathrm{~s}$ & $1.47 \times 10^{-2}$ & $1.99 \times 10^{-2}$ \\
\hline$\rho, \%$ & 8.5 & 11.9 \\
\hline
\end{tabular}

\section{Registro de parámetros ambientales}

Las Fig. 3 a 5 muestran el comportamiento de los parámetros ambientales (humedad relativa, temperatura y precipitación) durante los primeros meses de exposición.

La gráfica de barras de la Fig. 3 muestra que la humedad relativa promedio oscila entre el 80 y $85 \%$ durante esta etapa de estudio.

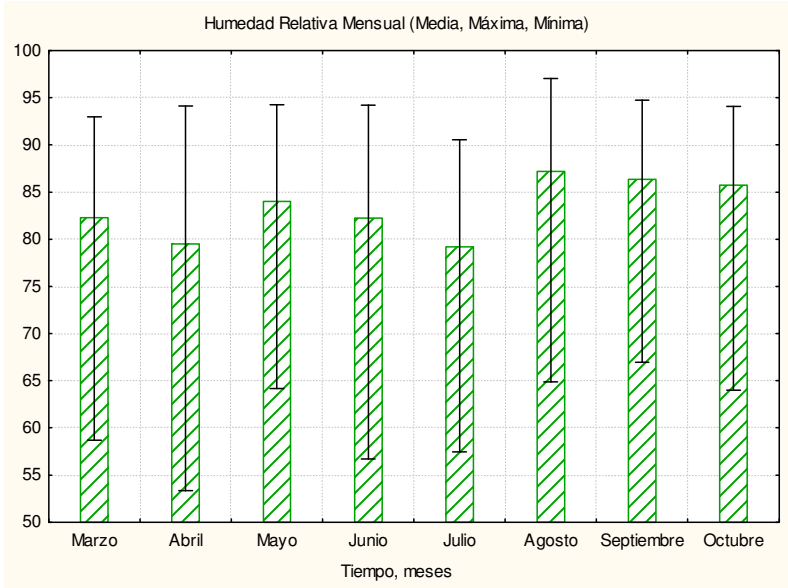

Figura 3. Variación mensual de la humedad relativa (2007).

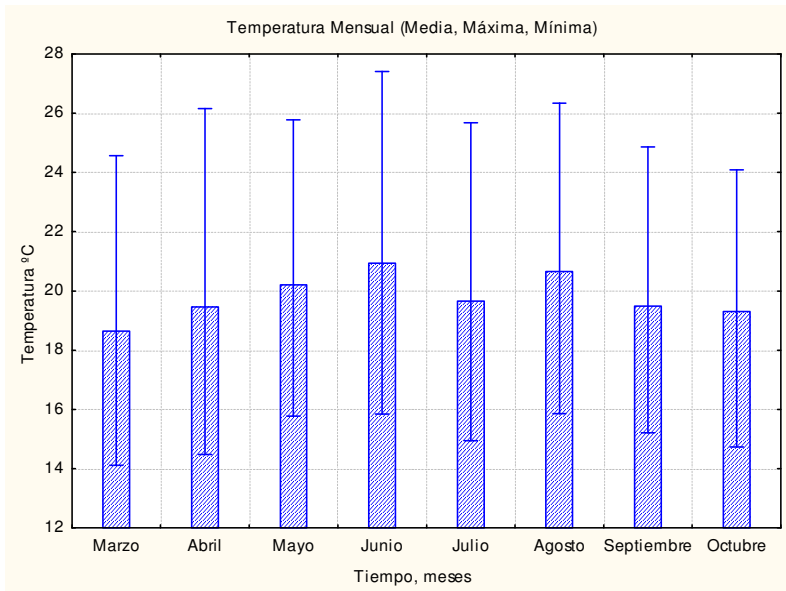

Figura 4. Variación mensual de la temperatura (2007). 
La temperatura promedio registrada osciló entre $18{ }^{\circ} \mathrm{C}$ y $21{ }^{\circ} \mathrm{C}$ (Fig. 4). El promedio mayor de precipitación se registró en el mes de septiembre (Fig. 5), alcanzando un valor de $7.5 \mathrm{~mm}$.

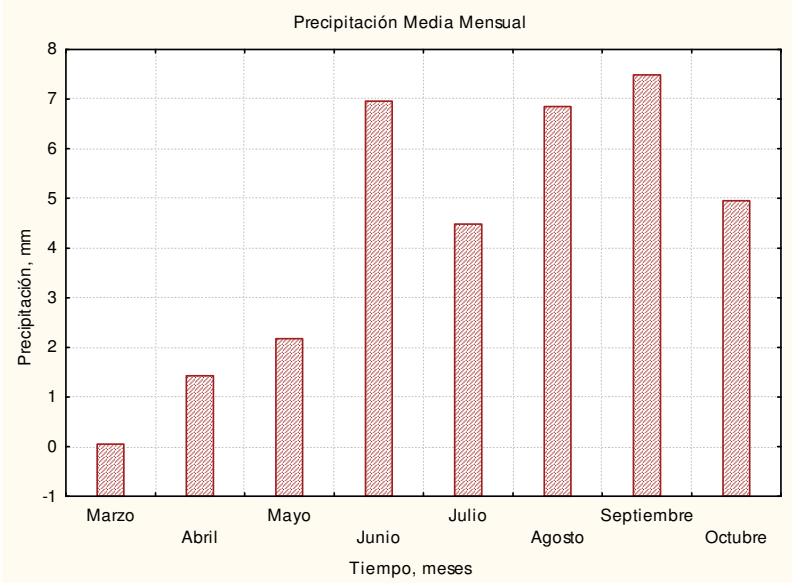

Figura 5. Gráfica de variación mensual de la precipitación pluvial (2007).

\section{Potencial de corrosión}

La interpretación de los valores de los potenciales de corrosión se hizo con base en la Norma ASTM C 876-91 [6] (ver Tabla 3).

Tabla 3. Potenciales de corrosión en hormigón reforzado.

\begin{tabular}{|l|l|}
\hline \multicolumn{2}{|c|}{$\begin{array}{l}\text { Potenciales de corrosión } \\
\text { Norma ASMT C 876-91 }\end{array}$} \\
\hline $\begin{array}{l}\text { Potenciales más positivos que } \\
-200 \mathrm{mV}\end{array}$ & $\begin{array}{l}\text { El riesgo de que ocurra } \\
\text { corrosión es del 10\% }\end{array}$ \\
\hline Si está entre -200 y -350 mV & se tiene una incertidumbre \\
\hline Si son más negativos & $\begin{array}{l}\text { la probabilidad de que se esté } \\
\text { que -350 mV }\end{array}$ \\
\hline
\end{tabular}

Los resultados de la medida de potenciales de corrosión se pueden observar en las Fig. 6 a 8, que corresponden a las seis vigas del estudio: tres de relación a/c = 0.45 y tres de 0.65 . Se presentan los resultados de la cara expuesta a los vientos predominantes.

En la Fig. 6 se observa, de acuerdo a la norma ASTM C 876-91, una tendencia general de los potenciales que indica un $10 \%$ de probabilidad de corrosión en todas las varillas y sus diferentes recubrimientos, así como la calidad de mezclas de hormigón. En la viga 1 se observa cómo la varilla de recubrimiento de $15 \mathrm{~mm}$ presenta los valores más negativos; para la viga 4 se observa la influencia del recubrimiento en los últimos dos meses de monitorización, presentando los recubrimientos de $15 \mathrm{~mm}$ y $20 \mathrm{~mm}$ los valores más negativos. 


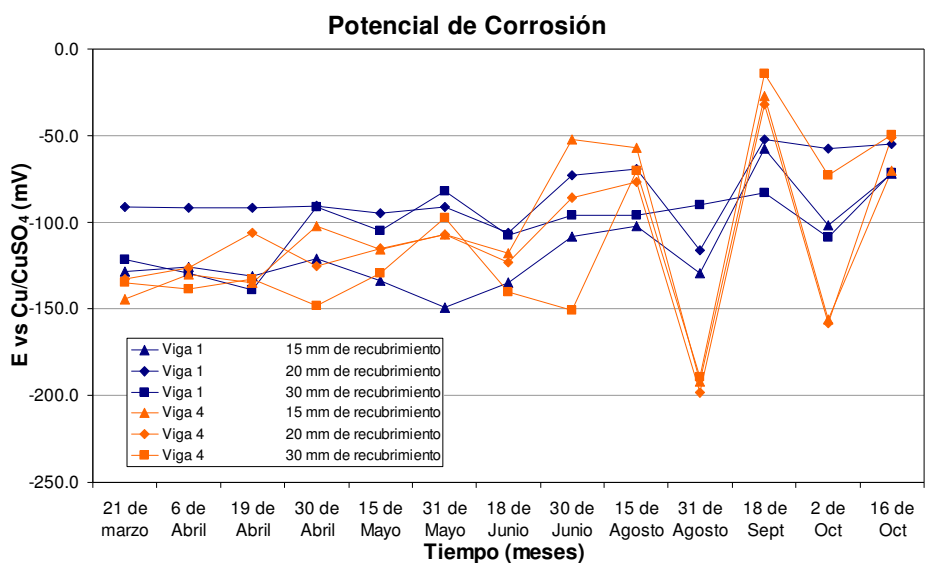

Figura 6. Comportamiento de los potenciales de corrosión. Viga 1 relación a/c $=0.45$ y Viga 4 relación a/c $=0.65$.

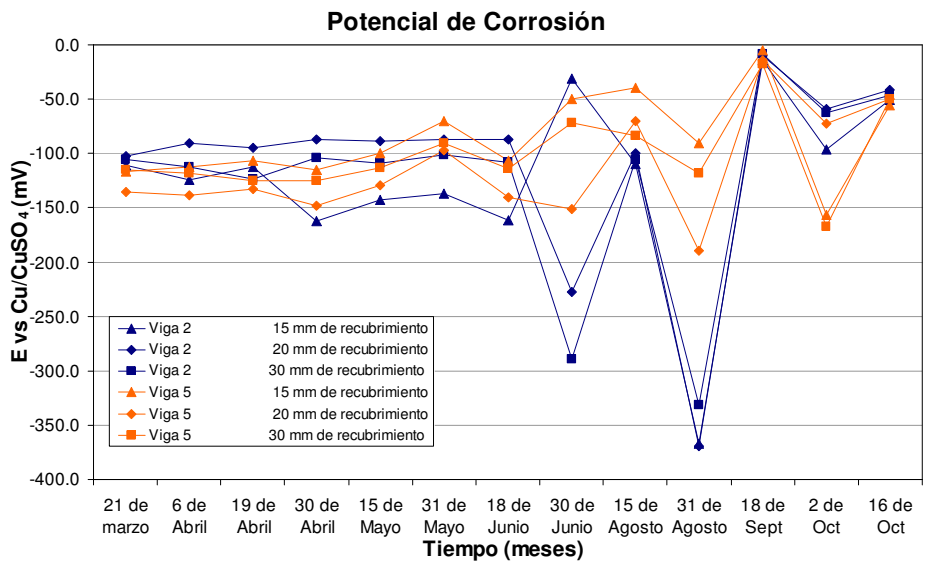

Figura 7. Comportamiento de los potenciales de corrosión. Viga 2 relación a/c $=0,45 \mathrm{y}$ Viga 5 relación a/c $=0,65$.

En la Fig. 7 se observa un comportamiento homogéneo de los potenciales en todos los recubrimientos, durante los primeros 3 meses. Asimismo, en la viga 2 se observa que el recubrimiento con valores más negativos es el de $15 \mathrm{~mm}$ y de la viga 5 el de $20 \mathrm{~mm}$, siendo más resistente el de $30 \mathrm{~mm}$ en ambas vigas. La tendencia general de los valores obtenidos indica, con base en la norma ASTM C-876-91, un 10\% de probabilidad de corrosión en todas las varillas y sus diferentes recubrimientos, así como la calidad de mezclas de hormigón. Se presentan dos lecturas (junio y agosto) que señalan la activación de los refuerzos de la viga 2, con valores que indican incertidumbre de la presencia de corrosión. Sin embargo, en los últimos dos meses dichos refuerzos vuelven a un estado de pasivación, mostrando valores más positivos que $-200 \mathrm{mV}$.

La Fig. 8 muestra valores de potencial que indican un $10 \%$ de probabilidad de corrosión, al igual que las Fig. 6 y 7. Al final de los meses de experimentación se observa la influencia de la relación a/c, ya que la viga $6(\mathrm{rel} . \mathrm{a} / \mathrm{c}=0.65)$ presenta valores de potencial que indican incertidumbre de que se esté dando corrosión. 


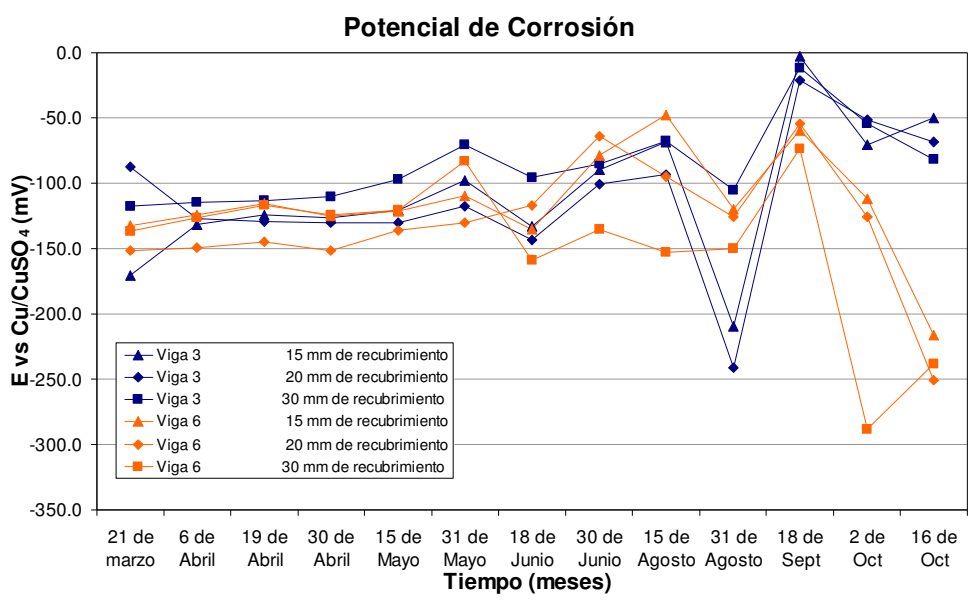

Figura 8. Comportamiento de los potenciales de corrosión. Viga 3 relación a/c $=0.45$ y Viga 6 relación a/c $=0.65$.

Las vigas 1,2 y 3 , se elaboraron con las mismas características para efectos de reproducibilidad, así como también para las vigas 4,5 y 6 .

\section{Cinética de corrosión}

La Tabla 4 presenta los valores de la cinética de corrosión del acero de refuerzo ( $\mathrm{i}_{\text {corr }}$ en $\mu \mathrm{A} / \mathrm{cm}^{2}$ ), de acuerdo a la viga analizada con sus correspondientes espesores de recubrimientos y calidad del hormigón. Se observa que en los especimenes de relación $\mathrm{a} / \mathrm{c}=0.45$, el acero que presenta menor cinética de corrosión es el que cuenta con un recubrimiento de $3.0 \mathrm{~cm}$, con un valor en promedio de $0.031 \mu \mathrm{A} / \mathrm{cm}^{2}$. Los especimenes 4 y 6 , de relación a/c $=0.65$, presentan valores de $i_{\text {corr }}$ similares en los tres recubrimientos. En general, todos los valores de la Tabla 4 indican, de acuerdo a la literatura, una magnitud de corrosión despreciable.

\section{Conclusiones}

Los valores de Ecorr que se observan a lo largo de estos ocho meses para los aceros de refuerzo con sus diferentes recubrimientos son, en general, más positivos que $-200 \mathrm{mV}$ lo que, con base en la norma ASTM C 876-91, indica un riesgo de corrosión del 10\%.

En las varillas de refuerzo de los especimenes de relación a/c $=0.45$ se distingue ligeramente la influencia del recubrimiento, presentando valores más nobles de Ecorr las varillas con recubrimiento de $3.0 \mathrm{~cm}$ y aún más negativos los de recubrimiento $1.5 \mathrm{~cm}$. Este comportamiento se identifica de igual forma en la cinética de corrosión, siendo el recubrimiento de $3.0 \mathrm{~cm}$ el de menor magnitud. En los especimenes de relación a/c $=0.65$ se observa cómo los valores de cinética o velocidad de corrosión son similares en los tres recubrimientos.

Hasta esta etapa de monitorización, los valores de la cinética de corrosión mostrados por los especimenes indican, de acuerdo a la literatura, un nivel de 
corrosión despreciable, concordando perfectamente con los valores de potenciales de corrosión.

Tabla 4. Valores de $i_{c o r r}$ para los primeros ocho meses de exposición; cara expuesta.

\begin{tabular}{|c|c|c|c|c|}
\hline \multirow[t]{2}{*}{ VIGAS } & \multirow[t]{2}{*}{ REC. (CM) } & $\begin{array}{c}21 \\
\text { /marzo/ } \\
07 \\
\end{array}$ & $\begin{array}{c}18 / \\
\text { junio/07 }\end{array}$ & $\begin{array}{c}21 / \\
\text { nov/07 }\end{array}$ \\
\hline & & $\begin{array}{c}\mathrm{i}_{\text {corr }} \\
\left(\mu \mathrm{A} / \mathrm{cm}^{2}\right)\end{array}$ & $\begin{array}{c}\mathrm{i}_{\text {corr }} \\
\left(\mu \mathrm{A} / \mathrm{cm}^{2}\right)\end{array}$ & $\begin{array}{c}\mathrm{i}_{\text {corr }} \\
\left(\mu \mathrm{A} / \mathrm{cm}^{2}\right)\end{array}$ \\
\hline \multirow{3}{*}{$\begin{array}{c}\text { VIGA } 1 \\
\text { Rel. a/c }=0.45\end{array}$} & 3.0 & 0.010 & 0.035 & 0.033 \\
\hline & 2.0 & 0.010 & 0.087 & 0.051 \\
\hline & 1.5 & 0.017 & 0.058 & 0.030 \\
\hline \multirow{3}{*}{$\begin{array}{c}\text { VIGA } 2 \\
\text { Rel. a/c }=0.45\end{array}$} & 3.0 & 0.019 & 0.043 & 0.033 \\
\hline & 2.0 & 0.025 & 0.039 & 0.051 \\
\hline & 1.5 & 0.016 & 0.073 & 0.041 \\
\hline \multirow{3}{*}{$\begin{array}{c}\text { VIGA } 3 \\
\text { Rel. a/c }=0.45\end{array}$} & 3.0 & 0.016 & 0.013 & 0.026 \\
\hline & 2.0 & 0.024 & 0.027 & 0.034 \\
\hline & 1.5 & 0.020 & 0.053 & 0.029 \\
\hline \multirow{3}{*}{$\begin{array}{c}\text { VIGA } 4 \\
\text { Rel. a/c }=0.65\end{array}$} & 3.0 & 0.008 & 0.025 & 0.016 \\
\hline & 2.0 & 0.015 & 0.032 & 0.030 \\
\hline & 1.5 & 0.020 & 0.016 & 0.022 \\
\hline \multirow{3}{*}{$\begin{array}{c}\text { VIGA 5 } \\
\text { Rel. a/c }=0.65\end{array}$} & 3.0 & 0.037 & 0.065 & 0.047 \\
\hline & 2.0 & 0.219 & 0.240 & 0.121 \\
\hline & 1.5 & 0.025 & 0.098 & 0.028 \\
\hline \multirow{3}{*}{$\begin{array}{c}\text { VIGA } 6 \\
\text { Rel. a/c }=0.65\end{array}$} & 3.0 & 0.019 & 0.063 & 0.023 \\
\hline & 2.0 & 0.014 & 0.028 & 0.020 \\
\hline & 1.5 & 0.017 & 0.025 & 0.020 \\
\hline
\end{tabular}

\section{Agradecimiento}

Se agradece a PROMEP el apoyo otorgado para la realización de este proyecto de investigación, autorizado por la SES mediante oficio no. PROMEP/103.5/07/2753, con cargo al "Proyecto de Apoyo a la Incorporación de Nuevos Profesores de Tiempo Completo PROMEP”.

\section{Influencia del recubrimiento sobre la cinética de corrosión en vigas de hormigón expuestas a la intemperie -Proyecto DURACON-}

\section{Resumen}

El presente trabajo forma parte del proyecto iberoamericano DURACON, el cual tiene por objetivo caracterizar la durabilidad de hormigones expuestos a las condiciones ambientales reinantes en Iberoamérica, mediante la exposición de especimenes de hormigón armado al menos en dos ambientes distintos, uno marino y uno urbano, en cada país participante. Los resultados obtenidos en los primeros ocho meses de exposición de los especimenes a las condiciones ambientales de la ciudad de Xalapa, 
México, son evaluados en este trabajo. Los especimenes se fabricaron con dos tipos de hormigones en función de la relación agua/cemento de 0,45 y 0,65. Se utilizó un mismo tipo de cemento y tres espesores de recubrimiento: 1,5, 2,0 y 3,0 cm. Las pruebas para evaluar la susceptibilidad a la corrosión del acero de refuerzo, fueron resistencia a la polarización lineal y monitorización de potenciales de corrosión siguiendo la norma ASTM C 876-91.

Palabras clave: Durabilidad, Corrosión, Hormigón, Medio Ambiente, Recubrimiento

\section{Referencias}

1. P. Castro, R. Castillo, J.J. Carpio, Corrosión en estructuras de hormigón armado, en Teoría, Inspección, Diagnostico, Vida Útil y Reparaciones, $1^{\mathrm{a}}$ edición IMCYC, México, 1998.

2. S. C. NMX-C-414-ONNCCE-2004, en Industria de la construcción Cementos hidráulicos - Especificaciones y métodos de prueba, Organismo Nacional de Normalización y Certificación de la Construcción y la Edificación, 2004.

3. Standard Test Method for Conducting Potentiodynamic Polarization Resistance Measurements. ASTM, in American Society for Testing and Materials, Philadelpia, USA, 1997, ASTM G 59 - 97.

4. O.T. Rincón, Manual de Inspección, Evaluación y Diagnóstico de Corrosión en Estructuras de Hormigón Armado. Durar, 1ª ed., Red Temática del CYTED, 1997, p. 200.

5. Standard Test Method for Compressive Strength of Cylindrical Concrete Specimens, in Annual Book of ASTM Standars, Philadelphia, 1992, Vol. 4.02, C39/C39M-04.

6. Standard Test for Half-Cell Potentials of Uncoated Reinforcing Steel in Concrete, in Annual Book of ASTM Standars, Philadelphia, 1992, Vol. 04.02, C876-91. 